\title{
DISCUSSION
}

\section{Creating virtual communities of practice for learning technology in higher education: issues and experiences}

\author{
Cathy Gunn* \\ University of Auckland, New Zealand
}

Dempster et al.'s paper (2003) describes an example of a community of practice that fits well with the definition given in current literature-one of a few that does not stretch the definition beyond the limits. Some would doubtless argue that communities evolve and cannot be created. This may point to a key element missing from the scenario described, i.e. a compelling reason for users to access the resources and participate in the communities provided for them by learning technologists.

Access to technology for educational use is a broader issue, as Burbules and Callister (2000) explain. In their definition, access involves having physical and operational capability, professional and cultural fluency and an appropriate conceptual model that allows competent interaction, innovation and creativity. This is still a long way off from many of the target users' current access levels.

The separation of the roles of expert, designer and intended user may be the root of the problem of mass participation identified by the authors. The disciplines of HCI and computer system design needed the involvement of professionals from other disciplines to overcome the barriers created by expert driven software engineering. So too, the mainstream use of technology in education may be awaiting the arrival of the lay practitioner in system specifications. To label this a problem is perhaps unfair, though. Experience tells us that designers of new technology rarely, if ever, foresee what their creation will eventually be used for or how long its infusion into practice will take.

If history provides a useful model, then the designer's role is to develop the opportunity for a critical mass of users to gain access and then devise their own useful

\footnotetext{
* Centre for Professional Development, University of Auckland, Private Bag 92019, Auckland, New Zealand. Email: ca.gunn@auckland.ac.nz
} 
purposes for the innovation. In this respect the RESULTS project team deserve high praise. Their approach to needs analysis has been systematic, inclusive and responsive. However, since users never know what they don't know, asking them what they want invariably yields results with limitations. What this questioning process doesextremely well in this case-is provide a sound basis for the development of a portal that reflects the needs and current capabilities of a range of practitioners in the field of learning technology. Presumably not, though, of the entire potential target user group, because only those with some experience of using learning technology may have been included in the research process. These needs analysis limitations relate back to the broader issues of access mentioned in the opening paragraph, and unavoidably exclude those potential users who currently do not have this capacity.

The debate about whether the RESULTS community was a core of specialists or a wider group is indeed a key question. If a 'dictionary definition' of a community of practice is applied, and particularly if the focus is on a learning community, it will surely involve a wider group so the less experienced can interact with and learn from the more experienced. Radiation of knowledge outwards from a core group of experts is a key learning opportunity, while interaction with less experienced users can provide understanding of the novice perspective as an input to design specifications.

The portal that was developed as a result of this research is one key element in an evolving landscape, where no one person or group can see the entire composition. It is another instance of the complex interaction of individually complex systems that inevitably leads to unexpected outcomes. As Edward Tenner (1996) reminds us, these outcomes are sometimes good, sometimes not, but they are usually unpredictable.

So if the RESULTS developers see a long way to go for the portal to enter mainstream use, perhaps they need to accept that they can guide the situation so far but not all the way. They have taken some very important steps in the right direction. The rest is up to the users. They want to support, though any amount of training and development still cannot add the final variable to the equation. Time is another essential ingredient in any significant culture shift, and that shift is what now needs to take place. There are many barriers to overcome - not all of them the responsibility of the developers.

One aspect (perhaps aspiration is a better term in this context) of a functional community is that the discourse of the discipline uses shared language with common understandings. It may be safe to assume that semantic differences are just one of many cultural variations that a learning technology portal will have to accommodate. A useful alternative to specific targeting of a range of differences is to simply acknowledge and attempt to accommodate them. Anything else may be too hard to achieve. In this respect, the paper does identify different categories of user and their preference for resource types. Academics prefer to have specific needs addressed while intermediaries want adaptability. This seems a logical reflection of roles and confirmation of the need for a range of different materials to suit a variety of users.

Another barrier RESULTS seems to have managed to overcome is the technical one. Most users do not and probably never will want to engage with the concepts of 
metadata and interoperability standards. In this case, those that do can and those that don't, don't have to. If the simplicity of the technical description in the paper reflects the visibility of technology to users of the portal then another important hurdle has been crossed. It may even have demystified this aspect for some readers.

In conclusion, the key remaining challenge underlies interpretation of the statement that 'RESULTS has been less successful in securing a critical mass of user participation'. Reasons are offered to explain the slow speed of uptake and possible remedies given - but here may be an important omission. The fact that 'it is not yet understood how people use portals' overlooks the complex nature of the evolving inter-relationship between expert and non-expert users and learning technology. It implies a need to understand existing practice better, although this does not reflect the reality of the innovation. The product of the synergy between current practice and new technology cannot be known at this stage of the integration process and is probably out with the control of the designers. While all the right steps have been taken to facilitate the process, there is a time when designers have to step back, be satisfied with their (excellent) creation and move on to the next task leaving users to make their own way. Now may not be the time to remove the training wheels but it will surely come. The required outcome is nothing short of a culture shift, upon which the political dimension (so lightly mentioned but so significant) will doubtless impact. Ultimately, it is up to the individuals who comprise the community to determine what the nature of this shift will be.

\section{References}

Burbules, N. \& Callister, T. (2000) Watch IT: the risks and promises of information technologies for education (Boulder, CO, Westview Press).

Dempster, J., Beetham, H., Jackson, P. \& Richardson, S. (2003) Creating virtual communities of practice for learning technology in higher education: issues, challenges and experiences, $A L T$ F, 11(3), 103-117.

Tenner, E. (1996) Why things bite back: technology and the revenge effect (London, Fourth Estate). 